\title{
Case Studies in Reconstruction Efficiency of Current Distribution in CICC's by Self Field Measurements
}

\author{
Yu. A. Ilyin and A. Nijhuis
}

\begin{abstract}
The measurements of the self magnetic field by means of Hall sensors (HS) in the vicinity of a superconducting cable-inconduit conductor (CICC) is often used to study current distribution effects. It is possible that current imbalance may affect the performance of CICC's and therefore knowledge of the current distribution is needed. Recently a model was presented to approximate the current distribution inside a conductor. Basically, the inverse problem must be solved in which the input data usually are the experimentally measured values of the local magnetic field, the location and orientation of the HS's and the geometry of the line or segment currents. All these, together with the adopted algorithm, determine the accuracy of the reconstruction procedure. In the present study the impact of two basic orientations of the HS: polar-symmetric and plane-parallel on the current reconstruction efficiency is performed for the analytical model developed in Twente. For the case study, a 36 strands CICC and a mock-up conductor are considered. The influence of the experimental errors and geometrical errors on the model output is also investigated.
\end{abstract}

Index Terms-Current imbalance, current reconstruction, self field measurements.

\section{INTRODUCTION}

A LREADY first attempts to investigate current imbalance in CICC's by using a set of self field measurements revealed the complexity of this problem [1]. It was possible to determine the location of the "apparent current center," but hardly possible to say anything about the magnitude of nonuniformity. Recently an interest to this problem has been regenerated and several works, both of analytical and experimental kind, were presented investigating this problem [2]-[7]. Nowadays an array of Hall sensors (HS) is becoming a regular tool in CICC's test programs. Self field measurements will be included in the test of the TFMC bus-bars (Toroidal Field Model Coil at FZK, Germany) and in the SExUP (Stability Experiment Upgrade at ENEA, Italy).

In [5] an identification algorithm to approximate the current distribution inside a CICC was reported. The goal of the present study is to validate this algorithm and to demonstrate its efficiency on a 36 strand CICC (prototype of the SExUP conductor) and with a mock-up conductor (similar to the ITER full size conductor).

The influence of the HS orientation on the reconstruction efficiency is also studied. In [7] quite extreme results in relation to the current distribution were found for the HS's in the high field region, all oriented parallel to the background field (plane-parallel configuration). Therefore it is necessary to clarify whether

\footnotetext{
Manuscript received August 5, 2002. This work was supported in part by the EU under Contract EFDA-00/552.

The authors are with the Department of Applied Physics, University of Twente, 7500AE Enschede, the Netherlands (e-mail: y.ilyin@tn.utwente.nl). Digital Object Identifier 10.1109/TASC.2003.812879
}

or not this geometry affects the efficiency in current reconstruction compared to HS's arranged in a polar-symmetric array (sensitive to only tangential or only radial self field components).

\section{THE MODEL}

\section{A. Theoretical Background}

For a reconstruction of the currents in strands or strand bundles of a superconducting cable from the set of self field measurements the solution of the inverse identification problem must be solved. Assuming linear material properties with magnetic field, the governing system of linear equations can be written in a matrix form as follows:

$$
\mathbf{G I}=\mathbf{B}
$$

where $\mathbf{B}$ is the $m$ element vector of the known experimental data (magnitudes of the self magnetic field and total transport current), $\mathbf{I}$ is the $n$ element unknown vector of current amplitudes and $\mathbf{G}$ is the $m$-by- $n$ matrix relating the currents to the measurement data. Matrix $\mathbf{G}$ depends on the geometry of the sources to be identified and on location and orientation of the magnetic probes (Hall sensors). When $m \geq n$ and $\operatorname{rank}(\mathbf{G})=n$ the problem is referred to as finding a least squares solution, i.e., to find the vector $\mathbf{I}$ that minimizes the norm $\|\mathbf{B}-\mathbf{G I}\|_{2}$. This solution always exists and is unique. When $m<n$ and $\operatorname{rank}(\mathbf{A})=m$, there are infinitive number of solutions $\mathbf{I}$ that exactly satisfy $\mathbf{B}-\mathbf{G I}=0$. In this case it is often useful to find the unique solution $\mathbf{I}$ that minimizes $\|\mathbf{I}\|_{2}$, and the problem is referred to as finding a minimum norm solution to an under-determined system of linear equations.

In the general case, when $\operatorname{rank}(G)<\min (m, n)$ (rank deficient problem) the unique solution is the minimum norm least squares solution $\mathbf{I}$ that minimizes both $\|\mathbf{B}-\mathbf{G I}\|_{2}$ and $\|\mathbf{I}\|_{2}$.

The solution of (1) can be written in most general form as:

$$
\mathbf{I}=\mathbf{G}^{+} \mathbf{B}
$$

where $\mathbf{G}^{+}$denotes a linear operator called pseudo inverse of $\mathbf{G}$ (if $\mathbf{G}^{-1}$ exists, then $\mathbf{G}^{+}=\mathbf{G}^{-1}$ and $\mathbf{I}=\mathbf{G}^{-1} \mathbf{B}$ ). In case of over-determined system, i.e., when $m>n$ and $\operatorname{rank}(\mathbf{G})=$ $n$, the linear operator $\mathbf{G}^{+}=\left(\mathbf{G}^{T} \mathbf{G}\right)^{-1} \mathbf{G}^{T}$. In case of underdetermined system, i.e., when $n<m$ and $\operatorname{rank}(\mathbf{G})=m$, the linear operator $\mathbf{G}^{+}=\mathbf{G}^{T}\left(\mathbf{G} \mathbf{G}^{T}\right)^{-1}$.

The problem of current reconstruction is very sensitive to errors in its description. Due to the physical nature of the problem, some experimental errors in $\mathbf{G}$ matrix or $\mathbf{B}$ input vector are unavoidable and can produce large errors in the solution I. Moreover, the accurate computation of the pseudo inverse is often difficult as it can be sensitive to round-off errors (and other small 


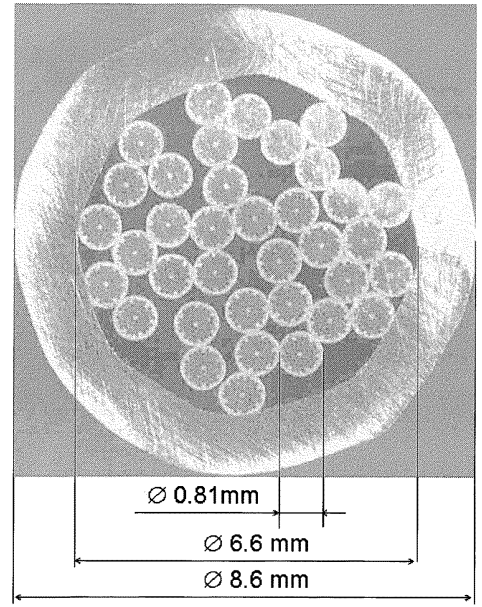

(a)

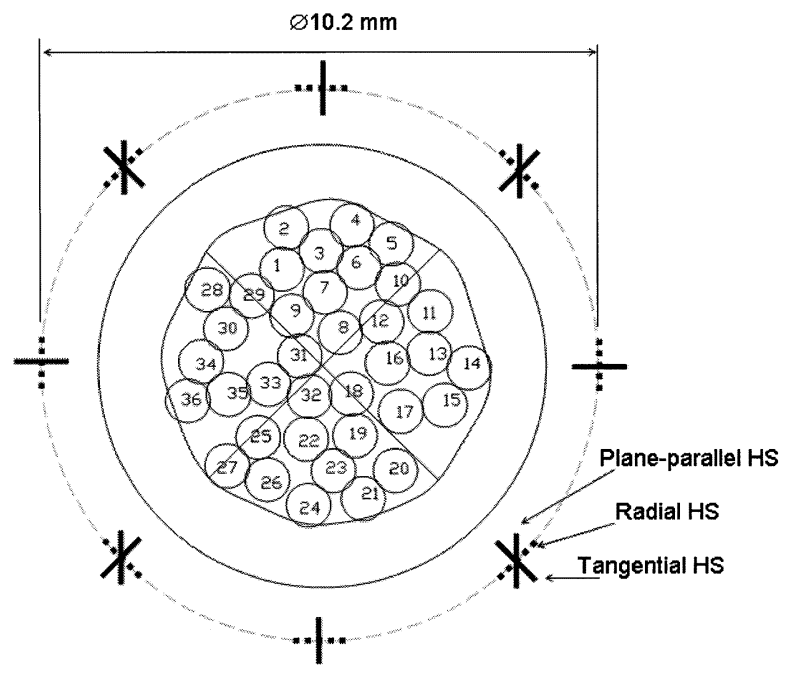

(b)

Fig. 1. Photo of the cross-section and corresponding layout of the 36 strands CICC used for current reconstruction. Strands are numbered from 1 to 36 . Each of the four imaginary segments includes approximately 9 strands. The HS's are arranged in three sets-tangential, radial and plane-parallel, each contains 8 HS's located at the same distance from the conductor's center.

changes in $\mathbf{G}$ ). In order to stabilize the solution, the SVD (singular value decomposition) technique can be utilized to calculate pseudo inverse. This technique is indispensable for ill-conditioned or rank deficient problems.

\section{B. Modeling 36 Strands Conductor}

This ITER related sub-size conductor is manufactured with CICC technology and consists of 36 strands embedded in round stainless steel jacket. The cabling pattern is $3 \times 3 \times 4$, the final stage sub-bundle consists of 9 strands. An example of the conductor's cross-section is shown in Fig. 1.

The task is to reconstruct the currents in each strand and final stage sub-bundles of the conductor from the limited number of self field magnitudes (which are, in turn, computed from an assumed current nonuniformity) and to compare the reconstruction efficiency by using different HS's arrangements.

For a first approximation the conductor is treated as straight and infinitively long with nontwisted isolated strands. The spatial location of the strands inside the jacket (radial and angular in relation to the conductor's center) was chosen according to con-

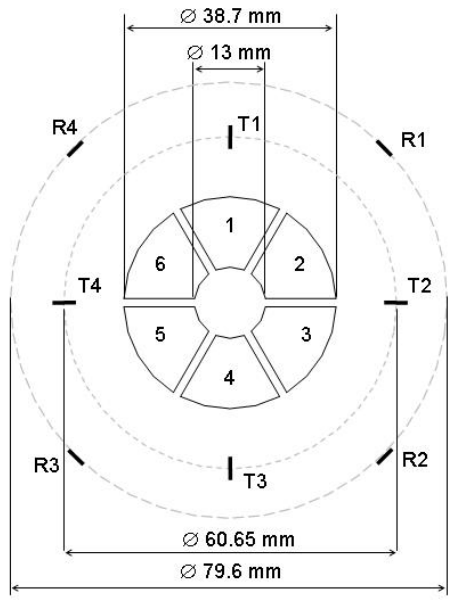

Fig. 2. Layout of the mock-up conductor, its geometry and position of the HS's used in the experiment.

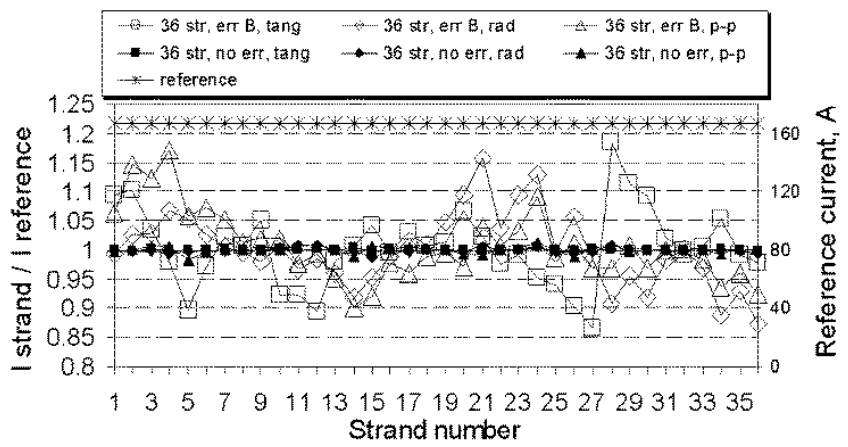

Fig. 3. Case of a uniform current distribution. Relative error in the current reconstruction caused by an error in the set of self field measurements. The connecting lines are for visual aid only.

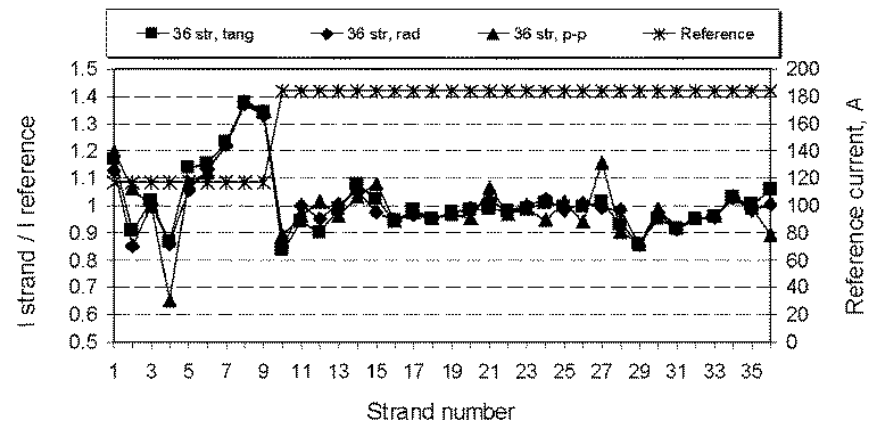

Fig. 4. Non-uniform current distribution on sub-bundle level in the 36 strands CICC. Relative error in the current reconstruction in each strand.

ductor cross-section (Fig. 1). As a practical case, the number of HS's is restricted to 8 , so 8 self field magnitudes are available as an input vector $\mathbf{B}$ for the model.

Three general geometries of the HS's orientations are considered (Fig. 1): all HS's are sensitive only to the tangential component of the self field (tangential HS's), only to the radial component (radial HS's) and to both components (plane-parallel HS's). For all cases the HS planes are parallel to the centerline of the conductor. The current density within each strand is assumed uniform over the cross-section and along the length of the strand. 
In case of 8 HS's and 36 strands, the corresponding system of equations is under-determined, and the unique solution is the minimum norm solution. From physical point of view this solution is "less extreme," which means that the calculated currents are as close as possible to the average current per strand (total current divided by the number of strands). In case of 8 HS's and 4 sub-bundles, the system is over determined, and the least squares problem must be solved.

\section{Modeling Mock-Up Conductor}

In order to validate the method of current reconstruction, the self field around a mock-up conductor was recently measured with a set of HS's at the Institute of Electrical Engineering of the Slovak Academy of Science [8]. The mock-up is a straight, half a meter long conductor assembled from 6 identical brass petals (Fig. 2). The petals are electrically insulated and it is possible to charge the petals individually with different currents to impose a certain well defined current nonuniformity. The dimensions of the mock-up conductor are similar to the dimensions of an ITER full-size conductor.

For current distribution analysis the self field measurements from 4 tangential HS's and 4 radial HS's are available. By using the data from only tangential HS's, only radial HS's or their combination as an input for the model, the currents in the petals can be found as a minimum norm solution of a linear least squares problem. The current density in each petal is assumed to be uniform over its cross-section and along the length of the petal.

\section{RESUlTS OF CURRENT RECONSTRUCTION}

\section{A. Case of 36 Strands Conductor}

The procedure of current reconstruction in the 36 strands CICC consists of two steps. First, at chosen currents (reference) in each strand, the magnitude of the self field components as sensed by the HS's in different orientations is calculated. At the second stage, the calculated values are used as an input for the model to reconstruct the currents. An example of the reconstruction results of a uniform current distribution at $6 \mathrm{kA}$ total current in the conductor is shown in Fig. 3. There the relative error in the current reconstruction (calculated to reference current ratio) is plotted for two cases-when there is no error introduced in the self field measurements and when there is a realistic absolute total error of, for example, $7 \mathrm{mT}$ in one of the self field magnitudes. In case when no error is introduced, the currents in each of 36 strands can be found with less than $1 \%$ error between calculated and reference values. Conversely there is up to $15 \%$ error in currents when the error is introduced for one of the HS.

Note, that absolute $7 \mathrm{mT}$ error means $3 \%$ relative error for tangential and more than $100 \%$ error for radial HS's.

In practice, an accurate measurement of the radial component is difficult because of its small magnitude ( $\sim 6 \mathrm{mT}$ at $6 \mathrm{kA})$ compared to tangential component ( $240 \mathrm{mT}$ at $6 \mathrm{kA})$. Also the influence of the "planar effect" on the HS output voltage can not be excluded in case of radial HS due to lager self field component parallel to a HS plane. An error in current reconstruction may arise not only from imprecise self field measurements, i.e., errors in vector $\mathbf{B}$, but also if the geometry of the current carrying elements (strands or sub-bundles) as well as geometry of the HS's is not known precisely (these are errors in matrix G).

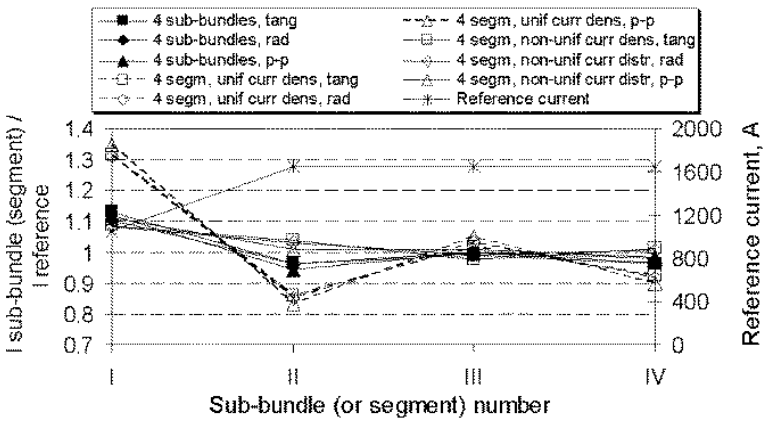

Fig. 5. Non-uniform current distribution on sub-bundle level in the 36 strands CICC. The relative error in the current reconstruction in each sub-bundle and in imaginary segments, assuming uniform or nonuniform current distribution over the segments, is plotted.

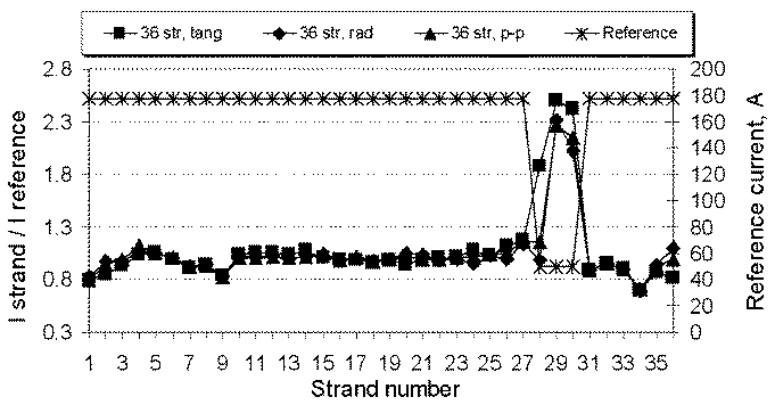

Fig. 6. Localized nonuniform current distribution in the 36 strands CICC. Relative error in the current reconstruction in each strand.

Obviously, the knowledge of the precise geometry and accurate self field measurements are essential for a current reconstruction. The following examples are based on the assumption of no errors in matrices $\mathbf{B}$ and $\mathbf{G}$.

In Fig. 4 the relative error in the reconstruction of currents is shown for the case of a nonuniform current distribution on the level of sub-bundles. The total current remains 6 kA. From Fig. 4 one can see that results from all three sets of HS's practically coincide. The maximum error between the calculated and measured currents reaches $40 \%$. However, an error between calculated and reference currents in each sub-bundle (as a sum of the strands currents) is within $10 \%$ (Fig. 5).

The currents in the sub-bundles were also found by using the four-segment model, in which each segment consists of 9 strands. The results are shown in Fig. 5. In this case the maximum error between calculated and reference currents increased up to $30 \%$.

This error can be substantially suppressed if the exact number of strands in each segment is taken into account. Then, this error drops to below $10 \%$.

In Figs. 6 and 7 the relative error in current reconstruction for a local nonuniform current distribution is shown for single strands and for sub-bundles correspondingly. In this case the maximum error between calculated and reference currents in the single strands increases dramatically. The relative error for a sub-bundle current reconstruction is within $10 \%$ if calculated as a sum of single currents and up to $20 \%$ as a result of a four segment model. (If the exact number of strands in each segment is taken into account, then the error of the four segment model drops back to $10 \%$.) It is important to notice that the results 


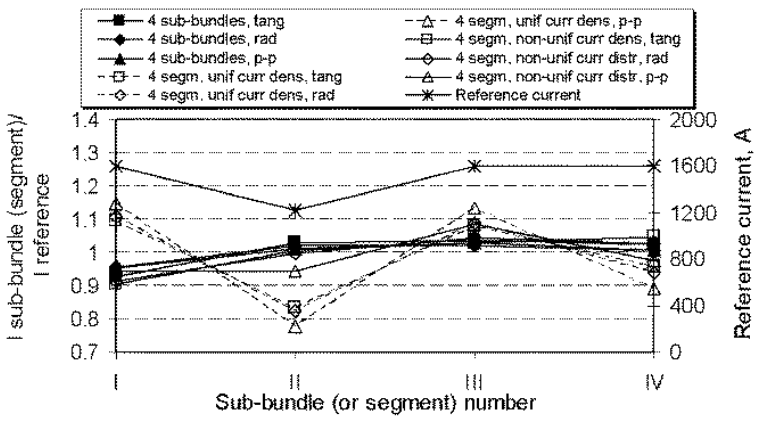

Fig. 7. Localized nonuniform current distribution in the 36 strands CICC Relative error in the current reconstruction in each sub-bundle and in imaginary segments assuming uniform or nonuniform current distribution over the segments.

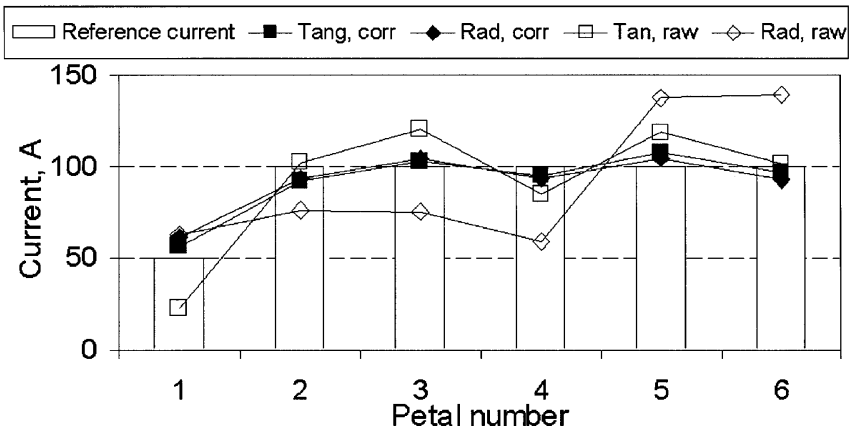

Fig. 8. Results of the currents reconstruction from the sets of measurements on the mock-up conductor with imposed current nonuniformity. Two methods are considered: the self field pattern corresponding to the uniform current distribution either is taken into account or not.

practically do not depend on which set of HS's is chosen for the current reconstruction as long as the measurement error can be neglected.

It appears that a reconstruction of the currents still has an acceptable accuracy for sub-bundles but is practically meaningless for individual strands because the strand locations are unknown.

\section{B. Case of Mock-Up Conductor}

An example of the current reconstruction from the set of self field measurements by four radial and four tangential HS's on the ITER full-size mock-up conductor is shown in Fig. 8. The current in one petal is $50 \mathrm{~A}$ and in five other petals it is $500 \mathrm{~A}$ (100 A per petal). In order to find the currents by using only 4 HS's, the minimum norm least squares solution of the under-determined system of equations must be found. If the "raw" experimental data are taken into account, then there is considerable deviation of the calculated currents from reference currents, especially for radial HS's. This error can be reduced if the self field pattern from the uniform current distribution is known. Such an experiment was performed and the self field at a uniformly distributed current of $600 \mathrm{~A}$ was measured. Then, using the difference between the self field magnitudes corresponding to the two current distributions and calculated self field at uniform current, the currents can be reconstructed with smaller error as shown in Fig. 8. It should be remarked that in this method the results from both sets of HS's coincide with each other. The difference between reference and computed currents does not exceed $10 \%$ when calculated by using the tangential component and is within $15 \%$ when calculated by using the radial component.

\section{SUMMARY}

In practice it mostly happens that the number of available HS's is less than number of current carrying elements. Thus, the corresponding system of equations is generally under-determined. For this case the unique solution can be found as a minimum norm least squares solution of under-determined system. Since the solution is very sensitive to experimental errors, the most effective way to avoid large errors is to determine (once) experimentally the self field pattern corresponding to a uniform current distribution.

The reconstruction of currents in each strand is unrealistic, especially in the case of local nonuniformity. However, the proposed model gives an acceptable solution for currents in subbundles or petals of the cable $( \pm 30 \%)$ but only if their (exact geometry and) location is known and all experimental errors are minimized.

For this reason it is attractive to subdivide the cable into subbundles but as a consequence it is assumed that the current in a bundle is homogeneously distributed. This method can give meaningless solutions when local nonuniformities are present inside the bundles.

The results from HS's in tangential, radial or plain-parallel arrays are similar if there is no input error introduced. However, measurements of the radial component are usually less accurate and this introduces a larger error in the reconstruction.

\section{ACKNOWLEDGMENT}

The authors are grateful to Dr. M. Polak and Dr. P. Ushak from the IEE of SAS for providing experimental data, and Dr. M. Ciotti from ENEA for providing the photo of the conductor.

\section{REFERENCES}

[1] V. S. Vystosky, M. Takayasu, and J. V. Minervini, "On the position of the apparent current center inside the CICC during external magnetic field ramp," Adv. Cryogenic Eng., vol. 42, pp. 1249-64, 1996.

[2] F. Bellina, P. Bettini, and F. Trevisan, "Analysis of the optimal location of magnetic field probes for the determination of the current distribution inside the s/c cables," IEEE Trans. Appl. Superconduct., vol. 11, pp. 2379-82, 2001.

[3] T. Bonicelli et al., "Superconductive cables current distribution analysis," in 22nd Symp. on Fusion Technology, Helsinki, Finland, Sept. 2002.

[4] P. Bruzzone, A. Formisano, and R. Martone, "Optimal magnetic probes location for current reconstruction in multistrand superconducting cables," IEEE Trans. Magn., vol. 38, p. 1057, 2002.

[5] A. Nijhuis, Yu. Ilyin, and H. H. J. ten Kate, "Analysis of the current distribution in the ITER CS-insert model coil conductor by self field measurements," IEEE Trans. Appl. Superconduct., vol. 12, pp. 1675-1679, Mar. 2002.

[6] Yu. Ilyin, A. Nijhuis, H. H. J. ten Kate, and P. Bruzzone, "Self field measurements by Hall sensors on the SeCRETS long sample CICC's in SULTAN," IEEE Trans. Appl. Superconduct., vol. 12, pp. 1667-71, Mar. 2002.

[7] Yu. Ilyin, A. Nijhuis, H. H. J. ten Kate, P. Bruzzone, and B. Stepanov, "Self field measurements by Hall sensors on the SeCRETS short sample CICC's subjected to cyclic load," , ASC 2002 COnf., paper 5LF04, to be published

[8] M. Polak, L. Jansak, J. Kvitkovic, P. Mozola, P. Usak, D. Erbenova, and J. Talapa, "Test results of the model measuring headTest results of the model measuring head," presented at the ITER Meeting, Padua, Italy, Jan. 2002. 